\title{
Models to assess the risk of introduction of selected animal viral diseases through the importation of live animals as a key part of risk analysis
}

\author{
Anna Gierak $^{1 凶}$, Krzysztof Śmietanka² \\ ${ }^{1}$ Department of Epidemiology and Risk Assessment, \\ ${ }^{2}$ Department of Poultry Diseases, National Veterinary Research Institute, 24-100 Puławy, Poland \\ anna.gierak@piwet.pulawy.pl
}

Received: July 16, $2021 \quad$ Accepted: December 7, 2021

\begin{abstract}
Introduction of an animal viral disease, especially a notifiable disease, into an importing country or region free from the disease may lead to serious epidemiological consequences and economic losses. Trade in live animals is historically considered one of the most important risk pathways. To estimate the magnitude of such risk, the likelihood of a virus' entry into a country and the consequences of this event should be jointly evaluated. Depending on data availability, the urgency of the problem and the detail level of the objectives, a risk assessment may be conducted in a qualitative, semi-quantitative or quantitative way. The purpose of this review was firstly to provide a brief description of each step of the risk analysis process, with particular emphasis on the risk assessment component, and subsequently to supply examples of different approaches to the assessment of the risk of the introduction of selected animal viral diseases. Based on the reviewed models, the overall likelihood of introduction of particular diseases was generally estimated as low. The output risk value was strongly dependent on the duration of the silent phase of the epidemic in the country of origin. Other parameters with some bearing upon the risk derived from the epidemiological situation in the country of origin and the biosecurity or mitigation measures implemented in the country of destination. The investigated models are universal tools for conducting assessment of the risk of introduction of various animal diseases to any country. Their application may lead to timely implementation of appropriate measures for the prevention of the spread of a disease to another country or region.
\end{abstract}

Keywords: infectious diseases, animals, import risk analysis, risk models.

\section{Introduction}

International trade in animals and their products is associated with a certain level of risk of introduction of an infectious animal disease into an importing country. Since the Agreement on the Application of Sanitary and Phytosanitary Measures (the SPS Agreement) came into force in 1995, countries which are members of the World Trade Organization (WTO) have been obliged to comply with its requirements, which are aimed at protecting human and animal health from such risk (36). The protective measures applied in a member country should be based on international standards, or if no such standards have been established or a higher level of protection is required, the measures should be dictated by science-based risk analyses. Such standards, guidelines and the framework for conducting an import risk analysis (IRA) are included in the World Organisation for Animal Health (OIE) Terrestrial Animal Health Code (the Code) (35).

The purpose of this review is to provide a brief, literature-based description both of the main steps of the risk analysis process with particular reference to risk assessment components and of examples of the application of the process to the risk of introduction of animal viral diseases into importing countries through the import of live animals.

\section{Import risk analysis}

Import risk analysis is a process helping to identify and manage the potential risk of infectious disease introduction associated with international trade in 
animals and their products. It should be conducted in a transparent manner and documented comprehensively at each step. Import risk analysis consists of the following components: hazard identification, risk assessment, risk management and risk communication. The relationship between these components is diagrammatised in Fig. 1.

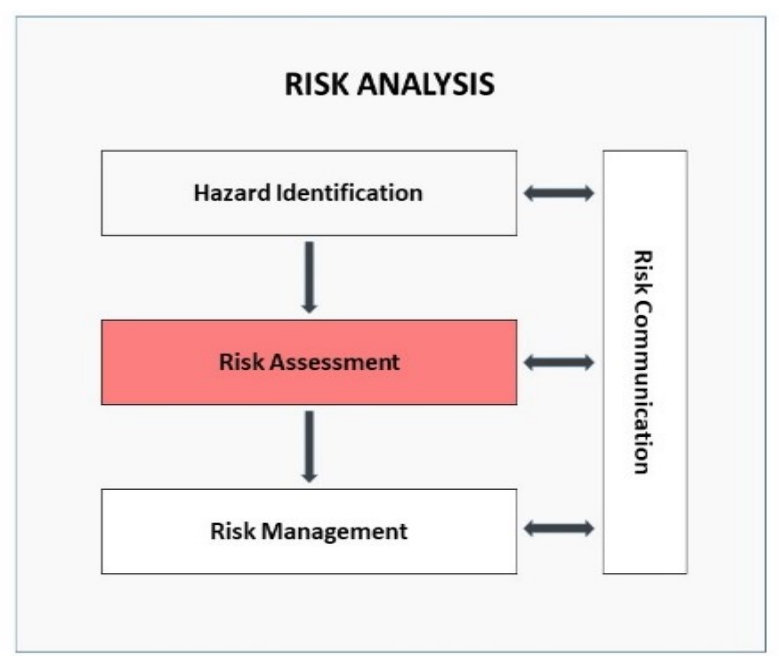

Fig. 1. The components of an import risk assessment. Source: World Organisation for Animal Health (OIE) Terrestrial Animal Health Code (35), modified

Hazard identification. This is the first step of the risk analysis process. Its purpose is to state if the importation of live animals is associated with a potential hazard using a dichotomous (yes/no) scale. It includes identifying the infectious agent and taking into account its various strains and variants and its capacity to cause infection in different animal species. Hazard identification requires knowledge of the epidemiological situation in an exporting country. Information on the hazards (including their occurrence and distribution) is available in the scientific literature, epidemiological studies and surveillance reports, especially in the case of notifiable diseases, of which each suspicion of occurrence is investigated and notified to the competent authorities of the destination country.

Risk assessment. In this step, the likelihood of the infectious agent's introduction into an importing country and the consequences are evaluated. This process is subdivided into four steps: (a) entry (release) assessment, (b) exposure assessment, (c) consequence assessment and (d) risk estimation. It is usually preceded by the schematising of the risk pathway depicting all possibilities of the infectious agent's introduction or indicating relevant risk factors. The relationship between the steps of the risk assessment process and how the steps correspond to stages on the pathway of infectious agent introduction from exporting to importing country is presented in Fig. 2.

Entry (release) assessment encompasses the description of the pathway through which the pathogen may be introduced into a particular animal subpopulation in an importing country. The pathway may be illustrated using a scenario tree. The probability may depend on various factors: biological (the species, age, and health condition of the animals), countryrelated (the pathogen's prevalence and the existence of vaccination, surveillance and control programs in the exporting country, e.g. testing and quarantine regimes) and commodity-related (the quantity of animals imported, possibility of contamination, and the impact of transport). Exposure assessment, in turn, spans the description of the pathway through which the animal population in an importing country may be exposed to an identified hazard and the probability estimation of this event. Various factors may influence the probability, such as the duration and frequency of exposure, properties of the infectious agent, presence of potential vectors, and animal demographics. Consequence assessment comprehends the description of consequences which may occur after the exposure of a particular subpopulation to the infectious agent and the probability of their occurrence. The potential consequences can be divided into those which are direct, such as the number of sick or dead animals and production losses, and those which are indirect, such as the cost of surveillance or implementation of additional preventive measures (e.g. treatment, vaccination, preventive culling, etc.) and the losses related to an export ban or export restrictions. Risk estimation comprises the results from all three of the above elements, which are used to evaluate the overall risk associated with the identified hazard.

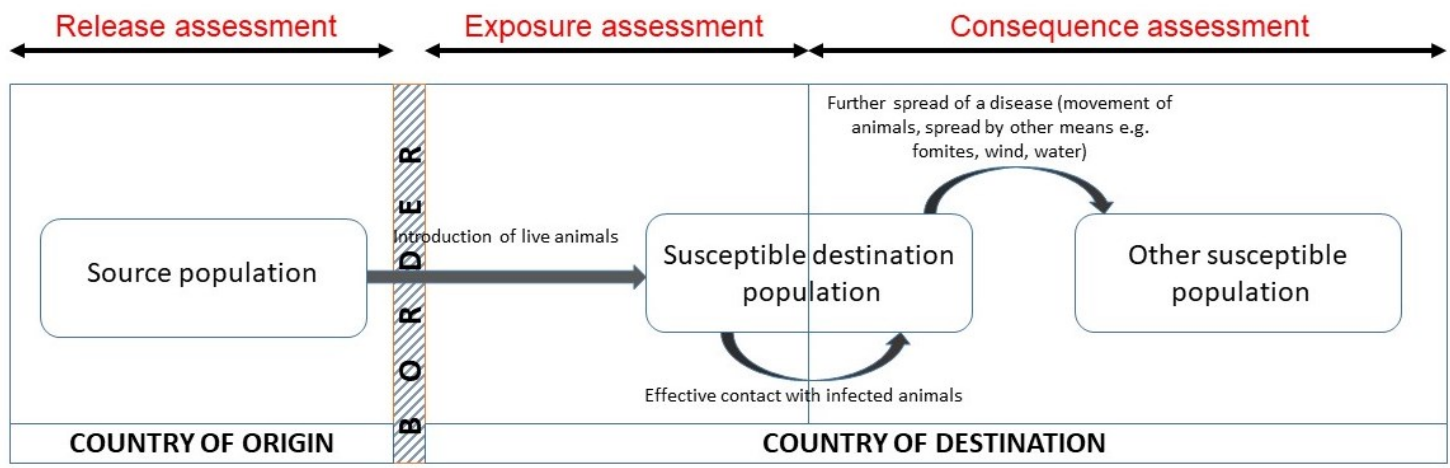

Fig. 2. The pathway of infectious agent spread overlaid with the steps of the risk assessment process. Source: Peeler and Taylor (26), modified 


\begin{tabular}{|c|c|c|c|c|}
\hline \multicolumn{5}{|c|}{ CONSEQUENCE } \\
\hline & Negligible & Low & Moderate & High \\
\hline High & Medium & High & High & Very high \\
\hline Moderate & Low & Medium & High & High \\
\hline Low & Low & Low & Medium & High \\
\hline Negligible & Negligible & Low & Low & Medium \\
\hline
\end{tabular}

Fig. 3. A risk matrix enabling the final level of risk to be defined by adjusting the probability of pathogen introduction by consequence severity. Source: Food and Agriculture Organization of the United Nations (12)

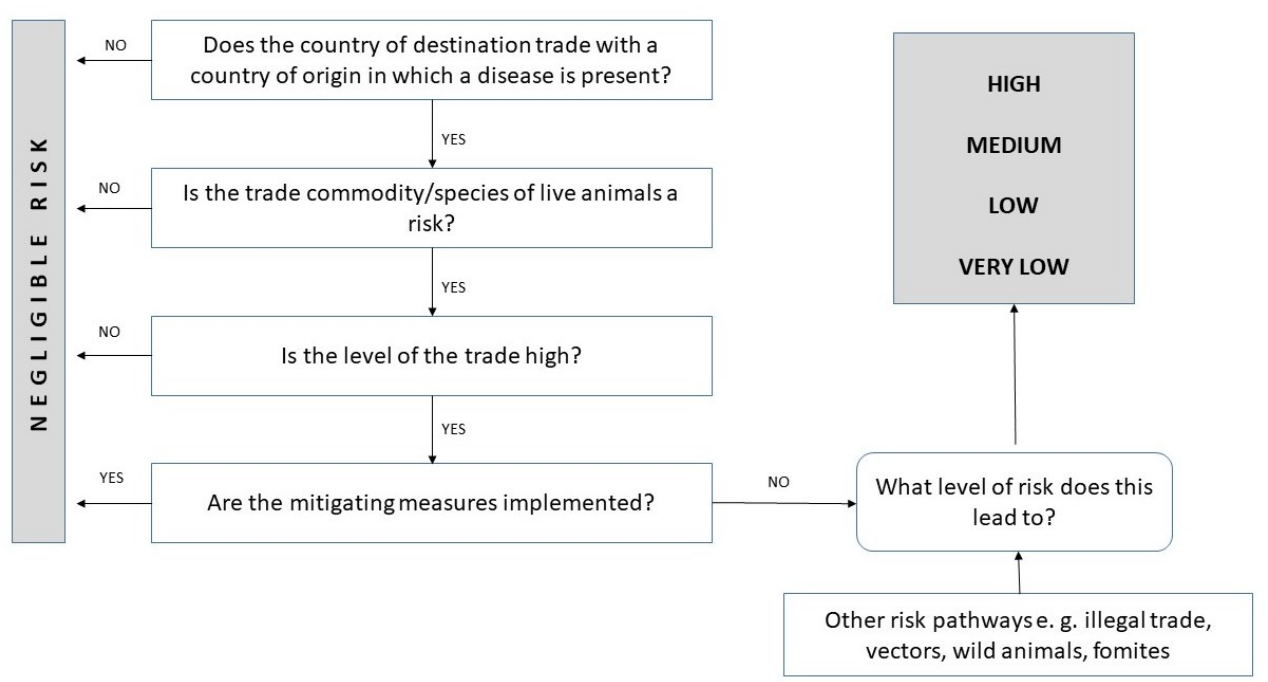

Fig. 4. An example of a flow chart for deciding if a disease poses a risk to the importing country. Source: Roberts et al. (27), modified

Various types of approach may be taken depending on the objectives, data availability and urgency of the questions under consideration. The model may be used for the assessment of either a single-pathogen or multiple-pathogen introduction. Single- or multiple-risk pathways may be considered. The risk may be assessed qualitatively, semi-quantitatively or quantitatively. In a qualitative risk assessment, both components of the risk, i.e. the likelihood of pathogen introduction and its consequences are described using the terms negligible, very low, low, moderate, high, or very high and subsequently both components are jointly evaluated (12) (Fig. 3).

In semi-quantitative risk assessment, values are assigned to qualitative estimates using weights or probability ranges and are subsequently combined using a mathematical operation. The output is expressed as a score. The model's structures are usually applicable to several infectious diseases. For instance, a tool developed by the UK Department for the Environment, Food, and Rural Affairs was suitable for rapid assessment of an infectious disease introduction risk via various risk pathways such as legal and illegal trade in live animals and animal products, transport (and fomites), and movement of vectors and wildlife. The final risk level is estimated by combining the scores obtained from answers to a set of questions which address the steps in the process of infectious agent introduction (27) (Fig. 4).
Quantitative risk assessment requires that a mathematical model be developed numerically linking all steps of the risk pathway (33). The magnitude of risk is also expressed numerically. This estimation may be deterministic (yielding a single output value such as an average, median, expected case, or worst case) or stochastic (giving a set of possible output values taken by the random variable). The basis for the development of the stochastic risk assessment model is a sequence of events expressed using conditional probabilities and subsequently computed according to the formula

$P(O)=P(I \cap X)=P(I) \cdot P(X \mid I)$

where:

$P(O)$ is the probability of disease outbreak in a destination country following the importation of animals,

$P(I)$ is the probability that at least one animal is infected when it crosses the border of a destination country

and $P(X \mid I)$ is the probability of exposure, transmission, infection and occurrence of disease in a destination country, given that at least one animal is infected when it crosses that country's border (22).

The epidemiological characteristics of the particular viral disease and the objectives of the risk assessment being conducted are not constants, and therefore the set of events included in a model may differ 
greatly from the generic model presented above. The extension of risk pathways through the addition of new elements or their reduction to the most significant part (the part generating the highest risk) is very common. The framework of these models assumes that the introduction probability follows a binomial process $(22,31)$. According to the OIE handbook (35), one-level and multilevel binomial probabilistic models are typically used for the estimation of the probability of an infectious agent's introduction into a country or region via importation of live animals. The main difference between these two types of model is the selection of animals for importation from the exporting country. In the one-level model, animals are selected from one single population (a country, region, or flock), in contrast to its multilevel counterpart, in which the population is subdivided into subsets (flocks or herds) and there is a two-stage selection, i.e. initially the herd is selected and then the animals within that particular herd are chosen (25). The probability of selection of at least one infected animal may be calculated according to the formula for one-level models

$$
P=1-(1-p)^{n}
$$

where:

$p$ is the prevalence of infection in the population and $n$ is the number of animals selected,

or the formula for multilevel models

$P=1-\left[1-H P\left(1-(1-p)^{n}\right)\right]^{h}$

where:

$H P$ is the herd level prevalence,

$p$ is the prevalence of infection in a herd,

$n$ is the number of animals selected from a herd and $h$ is the number of chosen herds.

As was shown, the one type of model can be implemented validly as an alternative to the other (31). Since slight differences in the outcomes of the aforementioned approaches were observed (the correlation between results from one- and multilevel models was $\mathrm{R}=0.94$ ), the implementation of the more comprehensive method is preferred when data concerning the number and distribution of animal herds in an exporting country is available. However, the application of the simpler method may be sufficient when the exact value of risk is not required.

As alternatives to using these two models, ordinal one-level and multilevel models can be applied to rank exporting countries by the risk that they pose to an importing country (31). The same ranking was obtained using a quantitative and an ordinal model, and the correlations between these two rankings were perfect at $\mathrm{R}=1$. The ranking may be computed through the pairwise comparison of the ratio between the values of $p$ and $n$ for the one-level model and $H P$ and $h$ for the multilevel model as follows:

If $p_{\text {country } 1, \text { country } 2}>1 / n_{\text {country } 1, \text { country } 2}$ country 1 poses a higher risk than country 2 .

If $p_{\text {country } 1, \text { country } 2}=1 / n_{\text {country } 1, \text { country } 2}$ country 1 poses the same risk as country 2 where: $p_{\text {country } 1, \text { country } 2}=p_{\text {country } 1 /} /$

$p_{\text {country } 2} ; n_{\text {country } 1, \text { country } 2}=n_{\text {country } 1} / n_{\text {country } 2}$. If $H P_{\text {country } 1, \text { country } 2}>1 / h_{\text {country } 1, \text { country } 2}$ country 1 poses a higher risk than country 2 .

If $H P_{\text {country } 1, \text { country } 2}=1 / h_{\text {country } 1, \text { country } 2}$ country 1 poses the same risk as country 2

where: $H P_{\text {country } 1, \text { country } 2}=H P_{\text {country } 1} /$

$H P_{\text {country } 2} ; h_{\text {country } 1 \text {, country } 2}=h_{\text {country } 1} / h_{\text {country } 2}$.

Risk management. The aim of the risk management process is to reduce the risk from the assessed level to an acceptable level through the application of sanitary measures. The measures chosen should be those which ensure the appropriate level of protection, i.e. the measures striking the best balance between minimising the possibility or at least the frequency of pathogen introduction and exerting negative effects on trade.

Risk communication. This is a continuous process which begins with hazard identification and is continued throughout all steps of the risk analysis process. During this process, information and opinions on both the potential hazard and the risk posed by it are gathered and the results of the conducted analysis are communicated to all stakeholders in the importing and exporting countries.

\section{Examples of IRA application}

Data sources. The information required for the definition or numerical description of model input parameters is obtained from various data sources such as: expert opinion, the OIE, the World Animal Health Information System, the Animal Disease Information System for disease outbreak occurrence data, the Trade Control and Expert System for trade volume data between countries of interest, the European Statistical Office, the Food and Agriculture Organization Corporate Statistical Database for agriculture data and livestock statistics, and scientific literature for diseaserelated information.

Risk models. The risk of introduction of several infectious viral diseases of animals into an importing country has already been assessed quantitatively using probabilistic models $(2,3,6,9,11,13,14,15,17,18$, $19,20,21,23,29,30,32,34)$. The step prior to the models' development was the formulation of the chain of events needed for the entry into and possible further spread within the specific population of the virus of interest in an importing country $(13,14,29)$. The events were expressed as probability distributions. The Monte Carlo method was used to generate draws from these distributions.

Most of the models were designed to assess the risk related only to the legal import of live animals; however, certain models combined it with other potential entry pathways. As an example, multiple-risk pathways were taken into account in the assessment of the risk of classical swine fever (CSF) introduction into the United 
States, Spain, Denmark and other selected EU member countries. Similarly, more than one pathway for African swine fever's (ASF) introduction into the United States and the Republic of Korea $(3,6,9,15,20)$ was incorporated into assessments. It was also considered in the risk assessment of the internal spread of foot and mouth disease (FMD) from areas at risk to those free of the disease in Argentina (17).

Not all the models included the full set of the three components of risk assessment, i.e. entry (release) assessment, exposure assessment and consequence assessment. Unrestricted risk assessment consisting only in the calculation of the probability of at least one infected animal reaching a country through international trade was performed in the majority of the models. This probability was assumed to follow a binomial process. The multilevel binomial model was used for the risk assessment of highly pathogenic avian influenza and Newcastle disease's introduction into Spain via legal import of live poultry $(29,30)$. This model was subsequently adapted to the assessment of the risk for Poland (13). The multilevel binomial model was also used to estimate the probability of low pathogenic avian influenza virus's (LPAIV) introduction into Poland and CSF's into Denmark $(3,14)$. The one-level binomial model, in turn, was applied to the risk assessment of the introduction of CSF and of several other viral infectious diseases such as Aujeszky's disease, FMD, and ASF $(6,9,15,17,18,19,20,21,23)$. As export is suspended at the moment of notification of a disease in a country or region, in general the models were developed under the assumption that the introduction of a virus is possible only during the silent phase of an epidemic, which is the time period from the first infection to the detection of the disease in the country of origin.

The estimated probability of a virus introduction into the countries where these modelling studies were carried out varied from negligible to high (in several cases). The movement of fattening and breeding pigs has proven to be the most likely $(<21 \%)$ route of Aujeszky's disease virus introduction into free areas in Spain (18). The probability of classical swine fever virus (CSFV) introduction into EU member states via import of pigs and pork products, returning livestock trucks and contact with wild boars was high for several countries: as an example, it was shown that the Netherlands can expect on average one CSF outbreak every 18 years in line with this multiple-risk pathway model (9). Introductions of CSFV into Spain through pig and wild boar movement, foot and mouth disease virus (FMDV) into the Malaysia-Thailand-Myanmar peninsula through livestock movement and LPAIV into Poland through importation of poultry were also likely scenarios and might cause one outbreak approximately every 9, 9 and 11 years, respectively $(14,20,34)$. In contrast, the probability of highly pathogenic avian influenza virus (HPAIV) introduction into Spain and Poland via poultry trade was almost negligible and one outbreak respectively every 735 and 326 years would be expected to occur in these two countries $(13,29)$. A very low probability of Newcastle disease virus' (NDV) introduction into Spain via this risk pathway would also be expected (on average one outbreak every 196 years) (30). Legal import of live pigs has not posed a risk of African swine fever virus' (ASFV) or CSFV's introduction into EU member states (one outbreak to be expected every 192 years) nor of these viruses' introductions into the United States (the estimated value of probability associated with the legal import of pigs and swine products corresponded to one outbreak of ASF every 276 and one of CSF every 201 years) $(15,23)$. Similarly, the probability of FMDV's introduction into Spain and the United States associated with importation of live animals was low (equivalent on average to one introduction every 40 and 241 years, respectively) as was the probability of this virus' introduction into new Argentinian regions associated with movement of animals within the country (one introduction every 169 years for sheep and goat movements and every 143 years for swine) $(17,19,21)$. The probabilities of bovine viral diarrhoea virus' (BVDV) introduction into the Netherlands through cattle imports and of BVDV and bovine herpesvirus 1's (BoHV-1) introduction into several regions within Spain through animal movements were, in turn, very heterogeneous in differing greatly between herd types $(2,32)$.

Among the input parameters of the models, some of the most influential were the ones associated with the epidemiological situation in the country of origin, such as inter- and intra-herd prevalence $(2,3,14,17,18,23)$ and the expected number of outbreaks before the detection of a disease $(3,23)$. The other weighty parameters were the ones associated with the mitigation of risk through the implementation of biosecurity measures in the destination country, such as quarantining animals $(2,15,19)$, serological testing (17) or disinfecting animal transport vehicles $(2,3,9)$. The disease-related parameters of length of the infectious period of a subclinically infected flock (14), probability of survival of an infected animal (23) and probability of the presence of detectable signs of infection during quarantine (34) also altered the introduction probability output value. This probability also differed greatly between different species of animals: for instance, animals carrying a virus asymptomatically posed a higher risk of introducing a disease because of the possibility of viral infection going undetected in their herd in the country of origin and subsequently during shipment or even the quarantine period.

The risk of infectious disease introduction was also assessed qualitatively (5). For example, the possibility of ASFV introduction into the United States and subsequent establishment in feral swine or native ticks was evaluated. Each of the potential routes of the virus' introduction was described and the designed prevention measures were outlined. According to this study, the routes of concern were the legal or illegal importation of 
live animals (or their products) and a bioterrorism event (4). The risk of CSFV introduction was assessed at regional level in the EU (8). To this end, all potential pathways of virus introduction into particular regions within the EU were taken into consideration and described using the pathway diagram. The conceptual framework for risk estimation comprising the relations between introduction, spread, prevention, control and expected economic losses was described. The results of the qualitative risk assessment suggest that movement of pigs during the incubation period of a disease, vehicles used for animal transport and human contacts are the main transmission paths (8). Transboundary spread of other pig diseases through international trade and travel was also depicted. The potential pathways of introduction and exposure were described. What was indicated as the main cause of a virus' introduction was the movement of infected animals from a country in which the presence of disease had not yet been confirmed and which still therefore officially had disease-free status. This may have led to the spread of a disease on a farm in a destination country or during slaughter (through inadequate barriers to fomite transmission) (1). Semi-quantitative rather than qualitative models were used to assess the risk of ASFV introduction into Finland and Belgium $(16,28)$. To this end, the NORA and Pandora rapid risk assessment tools were developed. Based on replies to a set of questions, emergence and consequence scores were prescribed and an overall risk score was calculated as their product. The assessed risk was low (a low probability of entry with a high severity of consequences) for Belgium and high (a high probability of entry with a high severity of consequences) for Finland. The highest score was for products of animal origin and for live animals. A generic risk assessment tool (including the outputs of two quantitative, three semi-quantitative and one qualitative model) was used to estimate the risk for the Netherlands. Based on the results derived from the models, the live animal trade pathway posed a higher risk to the Netherlands than to Finland (10).

Different IRA have been conducted using heterogeneous methods (7). Depending on the situation, either qualitative or quantitative approaches may be used and both types of method have been approved by the OIE. However, each has certain advantages and disadvantages. The quantitative methods, especially the stochastic ones, indisputably provide more precise and reliable output, given the frequent uncertainty and variability of input parameters. Typically, the models include a sensitivity analysis or test selected alternative scenarios (for example, the worst-case scenario), enabling both the identification of the most influential input parameters and the estimation of their impact on the output value of the introduction probability. It can be very useful, for instance, for exploring the impact of selected risk mitigation measures. However, the development of a quantitative model requires more data and is a time-consuming process. Another limitation of quantitative risk assessment models may be associated with rapid unnoticed changes in the epidemiological situation in an exporting country, which may lead to underestimation of the probability of a disease's occurrence in the country of origin. Particularly, because of the fact that many risk models were the most sensitive to the prevalence of infection. This type of quick change in epidemiological situation occurred in Lithuania in 2014 , when the export of 1,704 ready-for-slaughter pigs to Poland took place only two days before the detection of an ASF outbreak on the same farm (1). Similarly, before a ban on exportation was imposed, CSF spread from the Netherlands to Italy and Spain through the shipment of infected piglets in 1997 (1).

The qualitative and semi-quantitative methods may lead to arbitrary results, but they are simpler and may be more appropriate when a rapid estimation of risk is necessary. These methods may also be used as a preliminary evaluation of risk to decide if it should be measured with a more precise technique.

Conflict of Interests Statement: The authors declare that there is no conflict of interests regarding the publication of this article.

Financial Disclosure Statement: This study was funded by the "KNOW" (Leading National Research Centre) Scientific Consortium "Healthy Animal - Safe Food", Ministry of Science and Higher Education resolution no. 05-1/KNOW2/2015.

Animal Rights Statement: Not applicable.

\section{References}

1. Beltran-Alcrudo D., Falco J.R., Raizman E., Dietze K.: Transboundary spread of pig diseases: the role of international trade and travel. BMC Vet Res 2019, 15, 64, doi: 10.1186/s12917019-1800-5.

2. Benavides B., Casal J., Diéguez J.F., Yus E., Moya S.J., Armengol R., Allepuz A.: Development of a quantitative risk assessment of bovine viral diarrhea virus and bovine herpesvirus-1 introduction in dairy cattle herds to improve biosecurity. J Dairy Sci 2020, 103, 6454-6472, doi: 10.3168/jds.2019-17827.

3. Bronsvoort B.M. de C., Alban L., Greiner M.: Quantitative assessment of the likelihood of the introduction of classical swine fever virus into the Danish swine population. Prev Vet Med 2008, 85, 226-240, doi: 10.1016/j.prevetmed.2008.01.013.

4. Brown V.R., Bevins S.N.: A Review of African Swine Fever and the Potential for Introduction into the United States and the Possibility of Subsequent Establishment in Feral Swine and Native Ticks. Front Vet Sci 2018, 5, 11, doi: 10.3389/ fvets.2018.00011.

5. Brown V.R., Miller R.S., McKee S.C., Ernst K.H., Didero N.M., Maison R.M., Grady M.J., Shwiff S.A.: Risks of introduction and economic consequences associated with African swine fever, classical swine fever and foot-and-mouth disease: A review of the literature. Transbound Emerg Dis 2021, 68, 1910-1965, doi: 10.1111/tbed.13919.

6. Cho K.-H., Kim H.-J., Kim Y.-J., Kang H.-E., Martínez-López B., Lee J.-B.: Quantitative risk assessment of the African swine fever introduction into the Republic of Korea via legal import of live 
pigs and pig products. Transbound Emerg Dis 2021, 68, 385-396, doi: 10.1111 /tbed.13689.

7. De Vos C.J., Paisley L.G., Conraths F.J., Adkin A., Hallgren G.S.: Comparison of veterinary import risk analysis studies. Int J Risk Assess Manag 2011, 15, 330-348, doi: 10.1504/ijram. 2011.042672

8. De Vos C.J., Saatkamp H.W., Huirne R.B., Dijkhuizen A.A.: The risk of the introduction of classical swine fever virus at regional level in the European Union: a conceptual framework. Rev Sci Tech 2003, 22, 795-810, doi: 10.20506/rst.22.3.1433.

9. De Vos C.J., Saatkamp H.W., Nielen M., Huirne R.B.M.: Scenario tree modeling to analyze the probability of classical swine fever virus introduction into member states of the European Union. Risk Anal 2004, 24, 237-253, doi: 10.1111/j.0272-4332.2004.00426.x.

10. De Vos C.J., Taylor R.A., Simons R.R.L., Roberts H., Hultén C., de Koeijer A.A., Lyytikäinen T., Napp S., Boklund A., Petie R., Sörén K., Swanenburg M., Comin A., Seppä-Lassila L., Cabral M., Snary E.L.: Cross-Validation of Generic Risk Assessment Tools for Animal Disease Incursion Based on a Case Study for African Swine Fever. Front Vet Sci 2020, 7, 56, doi: 10.3389/fvets. 2020.00056 .

11. Fischer E.A.J., Martínez López E.P., De Vos C.J., Faverjon C.: Quantitative analysis of the probability of introducing equine encephalosis virus (EEV) into The Netherlands. Prev Vet Med 2016, 131, 48-59, doi: 10.1016/j.prevetmed.2016.07.005.

12. Food and Agriculture Organization of the United Nations: FAO Animal Production and Health Guidelines No. 24: Technical guidelines on rapid risk assessment for animal health threats. FAO, Rome, 2021, doi: 10.4060/cb3187en. http://www.fao.org/3/cb3187en/cb3187en.pdf.

13. Gierak A., Bocian Ł., Śmietanka K.: Risk Assessment of High Pathogenicity Avian Influenza Virus Introduction into Poland via Legal Importation of Live Poultry. Avian Dis 2015, 60, 178-182, doi: 10.1637/11081-040715-ResNote.

14. Gierak A., Śmietanka K., de Vos C.J.: Quantitative risk assessment of the introduction of low pathogenic avian influenza $\mathrm{H} 5$ and $\mathrm{H} 7$ strains into Poland via legal import of live poultry. Prev Vet Med 2021, 189, 105289, doi: 10.1016/j.prevetmed.2021.105289.

15. Herrera-Ibatá D.M., Martínez-López B., Quijada D., Burton K., Mur L.: Quantitative approach for the risk assessment of African swine fever and classical swine fever introduction into the United States through legal imports of pigs and swine products. PLoS One 2017, 12, e0182850, doi: 10.1371/journal.pone.0182850.

16. Kyyrö J., Sahlström L., Lyytikäinen T.: Assessment of the risk of African swine fever introduction into Finland using NORA-a rapid tool for semiquantitative assessment of the risk. Transbound Emerg Dis 2017, 64, 2113-2125, doi: 10.1111/tbed.12633.

17. Marcos A., Perez A.M.: Quantitative Risk Assessment of Footand-Mouth Disease (FMD) Virus Introduction Into the FMD-Free Zone Without Vaccination of Argentina Through Legal and Illegal Trade of Bone-in Beef and Unvaccinated Susceptible Species. Front Vet Sci 2019, 6, 78, doi: 10.3389/fvets.2019. 00078 .

18. Martínez-López B., Carpenter T.E., Sánchez-Vizcaíno J.M.: Risk assessment and cost-effectiveness analysis of Aujeszky's disease virus introduction through breeding and fattening pig movements into Spain. Prev Vet Med 2009, 90, 10-16, doi: 10.1016/j.prevetmed.2009.03.004.

19. Martínez-López B., Perez A.M., De la Torre A., Sánchez-Vizcaíno J.M.: Quantitative risk assessment of foot-and-mouth disease introduction into Spain via importation of live animals. Prev Vet Med 2008, 86, 43-56, doi: 10.1016/j.prevetmed.2008.03.003.

20. Martínez-López B., Perez A.M., Sánchez-Vizcaíno J.M.: A stochastic model to quantify the risk of introduction of classical swine fever virus through import of domestic and wild boars. Epidemiol Infect 2009, 137, 1505-1515, doi: $10.1017 / \mathrm{S} 0950268808001623$.
21. Miller G.Y., Ming J., Williams I., Gorvett R.: Probability of introducing foot and mouth disease into the United States via live animal importation. Rev Sci Tech 2012, 31, 777-787, doi: 10.20506/rst.31.3.2154

22. Morley R.S.: A model for the assessment of the animal disease risks associated with the importation of animals and animal products. Rev Sci Tech 1993, 12, 1055-1092, doi: 10.20506/rst.12.4.743.

23. Mur L., Martínez-López B., Martínez-Avilés M., Costard S., Wieland B., Pfeiffer D.U., Sánchez-Vizcaíno J.M.: Quantitative risk assessment for the introduction of African swine fever virus into the European Union by legal import of live pigs. Transbound Emerg Dis 2012, 59, 134-144, doi: 10.1111/j.18651682.2011.01253.x.

24. Murray N., MacDiarmid S.C., Wooldridge M., Gummow B., Morley R.S., Weber S.E., Giovannini A., Wilson D.: Handbook on Import Risk Analysis for Animals and Animal Products, Volume 1: Introduction And Qualitative Risk Analysis. OIE, Paris, 2004.

25. Murray N., MacDiarmid S.C., Wooldridge M., Gummow B., Morley R.S., Weber S.E., Giovannini A., Wilson D.: Handbook on Import Risk Analysis for Animals and Animal Products, Volume 2: Quantitative Risk Assessment. OIE, Paris, 2004.

26. Peeler E.J., Taylor N.G.H.: The application of epidemiology in aquatic animal health -opportunities and challenges. Vet Res 2011, 42, 94, doi: 10.1186/1297-9716-42-94.

27. Roberts H., Carbon M., Hartley M., Sabirovic M.: Assessing the risk of disease introduction in imports. Vet Rec 2011, 168, 447-448, doi: 10.1136/vr.d1784.

28. Roelandt S., Van der Stede Y., D'hondt B., Koenen F.: The Assessment of African Swine Fever Virus Risk to Belgium Early 2014, using the Quick and Semiquantitative Pandora Screening Protocol. Transbound Emerg Dis 2017, 64, 237-249, doi: 10.1111/tbed.12365.

29. Sánchez-Vizcaíno F., Perez A., Lainez M., Sánchez-Vizcaíno J.M.: A quantitative assessment of the risk for highly pathogenic avian influenza introduction into Spain via legal trade of live poultry. Risk Anal 2010, 30, 798-807, doi: 10.1111/j.15396924.2009.01351.x.

30. Sánchez-Vizcaíno F., Perez A., Lainez M., Sánchez-Vizcaíno J.M.: Quantification of the risk for introduction of virulent Newcastle disease virus into Spain through legal trade of live poultry from European Union countries. Avian Pathol 2010, 39, 459-465, doi: 10.1080/03079457.2010.520690.

31. Sánchez-Vizcaíno F., Perez A.M., Martínez-López B., Sánchez-Vizcaíno J.M.: Comparative Assessment of Analytical Approaches to Quantify the Risk for Introduction of Rare Animal Diseases: The Example of Avian Influenza in Spain. Risk Anal 2012, 32, 1433-1440, doi: 10.1111/j.1539-6924.2011.01744.x.

32. Santman-Berends I.M.G.A., Mars M.H., Van Duijn L., Van den Broek K.W.H., Van Schaik G.: A quantitative riskanalysis for introduction of Bovine Viral Diarrhoea Virus in the Netherlands through cattle imports. Prev Vet Med 2017, 146, 103-113, doi: 10.1016/j.prevetmed.2017.08.003.

33. Vose D.J.: Risk analysis in relation to the importation and exportation of animal products. Rev Sci Tech Off Int Epiz 1997, 16, 17-29, doi: 10.20506/rst.16.1.997.

34. Wongsathapornchai K., Salman M.D., Edwards J.R., Morley P.S., Keefe T.J., Van Campen H., Weber S.: Assessment of the likelihood of the introduction of foot-and-mouth disease through importation of live animals into the Malaysia-Thailand-Myanmar peninsula. Am J Vet Res 2008, 69, 252-260, doi: 10.2460/ajvr.69.2.252.

35. World Organisation for Animal Health (OIE): Terrestrial Animal Health Code, Section 2. Risk Analysis. Chapter 2.1. Import risk analysis. https://www.oie.int/index.php?id=169\&L=0\&htmfile= chapitre_import_risk_analysis.htm.

36. World Trade Organization: Agreement on the Application of Sanitary and Phytosanitary Measures. WTO, Geneva, 1995. http://www.wto.org/english/docs e/legal e/15-sps.pdf. 